\title{
LV Motion and Strain Computation from tMRI Based on Meshless Deformable Models
}

\author{
Xiaoxu Wang ${ }^{1}$, Ting Chen ${ }^{2}$, Shaoting Zhang ${ }^{1}$, Dimitris Metaxas ${ }^{1}$, \\ and Leon $\mathrm{Axel}^{2}$ \\ ${ }^{1}$ Rutgers University, Piscataway, NJ, 08854, USA \\ ${ }^{2}$ New York University, New York, NJ, 08854, USA
}

\begin{abstract}
We propose a novel meshless deformable model for in vivo Left Ventricle (LV) 3D motion estimation and analysis based on tagged MRI (tMRI). The meshless deformable model can capture global deformations such as contraction and torsion with a few parameters, while track local deformations with Laplacian representation. In particular, the model performs well even when the control points (tag intersections) are relatively sparse. We test the performance of the meshless model on a numeric phantom, as well as in vivo heart data of healthy subjects and patients. The experimental results show that the meshless deformable model can fully recover the myocardial motion and strain in 3D.
\end{abstract}

\section{Introduction}

The primary function of heart is mechanical pumping, and the strain fields are one of the basic measures of myocardial mechanics. The alteration of myocardial motion is a sensitive indicator of heart diseases such as ischemia and infarction. Usually infarcted myocardium and the myocardium adjacent to ischemia display abnormal motion pattern and smaller systolic strain. The motion and strain analysis can also contribute in the research on the development of some cardiac diseases, such as hypertrophy. In this paper, we will compare 3D strain field of normal hearts and hypertrophic hearts quantitatively.

Tagged Magnetic Resonance Imaging (tMRI) is a non-invasive way to track the in vivo myocardial motion during cardiac cycles. Compared to conventional MRI, tMRI provides more landmarks in myocardium. Myocardial motion in one direction can be quantitatively measured by tracking the deformation of tags that are initially in the perpendicular direction. In heart studies, usually tags are created in three sets of mutually orthogonal tag planes, two of which are perpendicular to the short axis (SA) image plane and one to the long axis (LA). Constructing a volumetric model with higher resolution from $2 \mathrm{D}$ tMRI slices can help with comprehensive understanding of myocardial motion and conducting quantitative analysis on 3D displacement fields and strain fields.

Spline models have been used to reconstruct cardiac motion [1] 2. Denny and McVeigh [3] gave a discrete finite difference analysis method to reconstruct displacement and strain fields. FEM models have been used on volumetric motion reconstruction. Young [4 built a cubic polynomial model driven by FEM, fit it

D. Metaxas et al. (Eds.): MICCAI 2008, Part I, LNCS 5241, pp. 636 644, 2008.

(C) Springer-Verlag Berlin Heidelberg 2008 
to the human tMRI data, and gave qualitative motion fields and strain fields. Deformable models have been used for the cardiac motion reconstruction from tagged MRI for years. Park et al. 5] presented deformable models combining spatially varying parameter functions to track the LV motion. Haber et al. 6] and Park et al. 7 further extended parameter functions to recover the right ventricle (RV) motion and conducted 4D cardiac functional analysis using Finite Element Methods (FEM).

As a comparably new technique tMRI is still under intensive research, and the imaging quality and resolution has been improved dramatically since it was firstly introduced. We propose a new meshless deformable model integrating meshless methods into the framework of deformable models developed by Metaxas et al $[8$. The meshless deformable model can model global motion pattern such as contraction and twisting and recover local deformation with intrinsic Laplacian representation. It also avoids time-consuming remeshing procedure required in FEM, which used as local deformation method in the previous cardiac deformable models. When the size of the finite elements is close to the scale of deformation, the elements tend to degenerate into irregular shape and cause singularity problem in numerical computation. As we increase the resolution of deformable meshes by incrementally subdividing elements into small size, element degeneration and remeshing become inevitable. With meshless methods, the remeshing can be replaced by a simple point-resampling procedure with much lower cost. Strain fields are calculated on the displacement fields with the Moving Least Square (MLS) method.

Our paper is organized as follows: section 2 introduces the framework of the new meshless deformable model; section 3 presents the deformation results on a numerical phantom and then elaborates its medical application on tagged MRI analysis; in section 4 we draw the conclusions.

\section{Methology}

\subsection{Data Description and Prepossessing}

Tagged MR images were obtained from a Siemens Trio 3T MR scanner with 2D grid tagging. The 3D tagged MR image set we used consisted of a stack of $5 \mathrm{SA}$ image sequence equally spaced from the base to the apex of the LV, and $3 \mathrm{LA}$ images which are parallel to the LA and with 60 degree angles in between, as shown in Figure 1 1 (a).

The LA and SA tag MR images were aligned with the spatial information in the dicom header file. The heart wall around the LV is segmented semiautomatically using a machine-learning based approach as in 9 . We detected hundreds of landmarks on the myocardial contours based on local curvature. The landmarks were then matched between image contours and the corresponding slices of the model. The matched point pairs provided long range external forces for the convergence of the meshless deformable model and the image data. The boundary of the registered heart is displayed in Figure 1(b). 

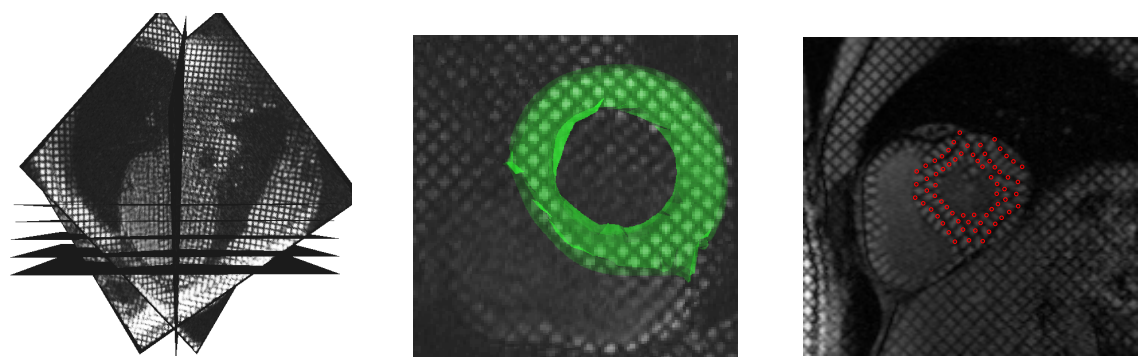

Fig. 1. (a)The setting of MR Images: 5 SA parallel images are placed with equal space from apex to base. 3 rotated LA images are taken with 60 degree angles in between. (b)Registered LV on SA images. (c) The intersections of grid tagging lines tracked by gabor filters.

The automatic tracking of tag intersections provided the external forces for the meshless deformable model. As introduced in Chen et al. 10]11, a Gabor filter bank was implemented to generate corresponding phase maps for tMRI images. A Robust Point Matching (RPM) module has been integrated into the approach to avoid false tracking results caused by through-plane motion and irregular tag spacing. Tracked tag intersections are shown in Figure 1(c).

\subsection{Motion Reconstruction by Meshless Deformable Models}

Different from previous works on LV motion reconstruction with deformable models, an object is represented as a point cloud inside the object boundary in meshless deformable models. The interaction of points are mechanized with radius based kernels. A point and its neighboring points are grouped into a phyxel with a kernel function.

Global Deformation. An object is represented by parameterized point clouds in meshless deformable models. The world coordinates of model points are transformed to model centered polar coordinates and controlled by a set of global parameters. The coordinates of points in the world coordinate system are transformed into a model-centered coordinate system as $x=c+R p$, where $c$ is the world coordinates of the model centroid, and $R$ is the rotation matrix. Modelcentered coordinates $p=s+d$ can be further decomposed into two parts to incorporate global and local deformations, which will be introduced in the following two subsections. The contraction and torsion of LV myocardium can be taken as global deformation. We interpret the model centered coordinates $s=f\left(q_{s}\right)=f\left(a_{0}, a_{1}, a_{2}, a_{3}, \tau\right)$ in a polar geometry with coordinates $(\alpha, \beta, w)$.

$$
e=w a_{0}\left(\begin{array}{c}
a_{1} \cos (\alpha) \cos (\beta) \\
a_{2} \cos (\alpha) \sin (\beta) \\
a_{3} \sin (\alpha)
\end{array}\right), \quad s=\left(\begin{array}{c}
e_{1} \cos (\varphi)-e_{2} \sin (\varphi) \\
e_{1} \sin (\varphi)+e_{2} \cos (\varphi) \\
e_{3}
\end{array}\right)
$$


Parameters $q_{s}$ include a scaling factor $a_{0}$, radiuses in three directions $a_{1}, a_{2}, a_{3}$, and a twisting factor $\tau$. In the LV reconstruction, usually we define $\alpha \in\left[-\frac{\pi}{2}, \frac{\pi}{4}\right]$ runs from apex to the base. $\beta \in[-\pi, \pi)$ is horizontal, starting and ending at the inferior junction. The transmural factor $w \in[0,1]$ is defined in a way that it equals to 1 on model's epi-surface, and 0 at model's centroid. Twisting angle $\varphi=\pi \tau \sin (\alpha)$.

We calculate the displacements of points by integrating velocities over time. The velocity can be derived from external force by dynamics equation

$$
\dot{x}=f
$$

The global deformation of the model is captured by applying small displacements on global parameters. The velocity of points can be deduced from the velocity of global parameters via Jacobian matrix $\mathbf{L}$.

$$
\dot{x}=\mathbf{L} \dot{q}_{s}
$$

The dynamics equation 2 can also be used on global variables $q_{s}$. The velocity of global variables $q_{s}$ can be calculated by combining formula 2 and formula 3 and applying the Lagrangian equation. The external forces on global parameters $f_{q_{s}}$ are integrated over the object volume

$$
\dot{q}_{s}=f_{q_{s}}=\int_{\Omega} f \mathbf{L}
$$

The integration over the volume can be interpreted as the sum of the integrals over each phyxel in the volume.

Local Laplacian Editing. We encode each point in the meshless deformable models into a Laplacian representation to keep the intrinsic geometric detail of myocardium. The Laplacian of a mesh is enhanced to be invariant to locally linearized rigid transformations and scaling in Sorkine et al. [12]. We further extend it from a surface editing tool to a method for tracking geometric details of a volume.

The geometry of points in the model can be described as a set of differentials $\Delta=\left\{\delta_{i}\right\}$. The Laplacian coordinate of a point as introduced in Desbrun et al. ([13]), is the difference between and the average of its neighbors.

$$
\delta=\mathscr{L}\left(x_{0}\right)=x_{0}-\frac{1}{d} \sum_{\left|x-x_{0}\right|<h} x
$$

The transformation can be described in a matrix form $\Delta=L X$, where $L=$ $I-D^{-1} A$. $A$ is the mesh adjacency matrix and $D=\left\{d_{1}, d_{2}, \ldots, d_{n}\right\}$ is the degree matrix. We combine landmarks and sampled points together to make a point set. Fixing the landmarks at the target locations $\left\{v_{i}\right\}$ obtained from the next MRI frame, the rest free points deform to minimize the following error function.

$$
E\left(X^{\prime}\right)=\sum_{i=1}^{n}\left\|T_{i} \delta_{i}-\mathscr{L}\left(x^{\prime}\right)\right\|^{2}+\sum_{i=1}^{m}\left\|x_{i}^{\prime}-v_{i}\right\|^{2}
$$


where transformation $T_{i}$ on each point is the unknown matrix and can be written as a linear function of $X^{\prime} . X^{\prime}$ can be solve by minimize the quadratic function. As a $3 \mathrm{D}$ transformation matrix with only rotation and uniform scaling on homogeneous coordinate, $T_{i}$ can be written as

$$
T_{i}=\left(\begin{array}{cccc}
s & -h_{3} & h_{2} & t_{x} \\
h_{3} & s & h_{1} & t_{y} \\
-h_{2} & h_{1} & s & t_{z} \\
0 & 0 & 0 & 1
\end{array}\right)
$$

Let vector $\left(s_{i}, \mathbf{h}_{\mathbf{i}}^{T}, \mathbf{t}_{\mathbf{i}}{ }^{T}\right)^{T}$ be the unknowns in $T_{i}$. The first term for each point in equation (6) can be rewrite as $\| A_{i}\left(\left(s_{i}, \mathbf{h}_{\mathbf{i}}{ }^{T}, \mathbf{t}_{\mathbf{i}}{ }^{T}\right)^{T}-b_{i} \|^{2}\right.$, where

$$
A_{i}=\left(\begin{array}{ccccccc}
x_{k_{1}} & 0 & x_{k_{3}} & -x_{k_{2}} & 1 & 0 & 0 \\
x_{k_{2}}-x_{k_{3}} & 0 & x_{k_{1}} & 0 & 1 & 0 \\
x_{k_{3}} & x_{k_{2}} & -x_{k_{1}} & 0 & 0 & 0 & 1 \\
\vdots & & & & & &
\end{array}\right), \mathbf{b}_{i}=\left(\begin{array}{c}
x_{k_{1}}^{\prime} \\
x_{k_{2}}^{\prime} \\
x_{k_{3}}^{\prime} \\
\vdots
\end{array}\right), k \in i \cup \operatorname{Neighbor}(i)
$$

The above least-squares problem can be solved by

$$
\left(s_{i}, \mathbf{h}_{\mathbf{i}}^{T}, \mathbf{t}_{\mathbf{i}}^{T}\right)^{T}=\left(A_{i}^{T} A_{i}\right)^{-1} A_{i}^{T} \mathbf{b}_{i}
$$

As long as $T_{i}$ is solved, we can update $X_{i}^{\prime}=T_{i} X_{i}$ accordingly. When $X^{\prime}$ converge, this error minimization problem is solved.

Transformation $T_{i}$ is an approximation of the isotropic scaling and rotations when the rotation angle is small. In our model, the major rotation is handled in the global deformation part. The small rotation angle of local deformation fits the small angle assumption of Laplacian edition.

\subsection{Strain Computation by Moving Least Squares}

After the displacements of points are computed, we want to compute the strain tensor at each point. Without a point set as a structured $3 \mathrm{D}$ grid, the strain tensor cannot be obtained by the definition. The deformation gradient is approximated with MLS (Lancaster and Salkauskas [14]). The MLS minimized the weighted difference between the observed displacement of a point and the displacement approximated by its neighbors with first order accuracy

$$
e=\sum_{j}\left(\tilde{u}_{j}-u_{j}\right)^{2} w_{i j}, \text { where } \tilde{u}_{j} \text { is } u_{j} \text { 's neighbor }
$$

Components of the displacement gradient $\nabla u$ at node $i$ can be computed as (for example, the $x$ component):

$$
\left.\nabla u\right|_{x_{i}}=A^{(-1)} \sum_{j}\left(u_{x}(j)-u_{x}(i)\right) x_{i j} w_{i j}, \text { where } A=\sum_{j} x_{i j} x_{i j}^{T} w_{i j}
$$


Given the initial position of a phyxel $x_{0}=(x, y, z)$ in a world coordinate and the displacement $u(t)=\left(u_{x}, u_{y}, u_{z}\right)$ at time $t$, the current position of the phyxel in the deformed model is $x(t)=x_{0}+u(t)$. The Jacobian of this mapping is

$$
J=I+\nabla u^{T}=\left[\begin{array}{ccc}
1+u_{x, x} & u_{x, y} & u_{x, z} \\
u_{y, x} & 1+u_{y, y} & u_{y, z} \\
u_{z, x} & u_{z, y} & 1+u_{z, z}
\end{array}\right]
$$

Given the Jacobian $J$, the Lagrangian strain tensor $\varepsilon$ of the phyxel is

$$
\varepsilon=\frac{1}{2}\left(J^{T} J-I\right)=\frac{1}{2}\left(\nabla u+\nabla u^{T}+\nabla u \nabla u^{T}\right)
$$

\section{Experimental Result}

\subsection{Test on a Phantom}

We tested the meshless deformable model and MLS strain computation with a numeric phantom. To test the performance of the meshless deformable model with sparse external forces, we reconstruct the motion using $10 \%$ of control points. The model still converges to the target state in the same accuracy. The strain field computed based on the deformation results are displayed in Figure 2 . Given a phantom in the similar size of LV, the MAE of the strain calculated by MLS is 0.0076 .
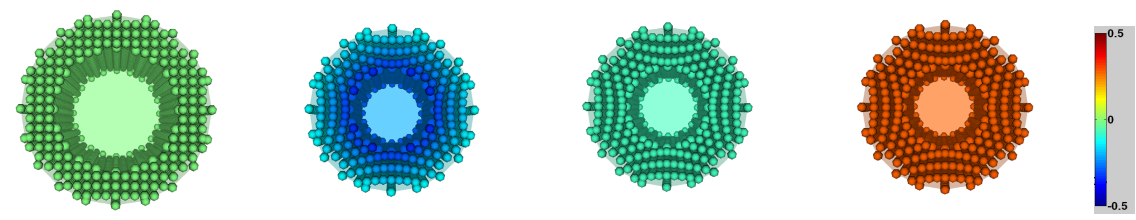

Fig. 2. The top view of strain field on a phantom(from left to right): initial, circumferential strain, longitudinal strain and radial strain

\subsection{Deformation and Strain Analysis}

After getting the deformation of the LV with meshless methods, we compute strain based on the deformation. Some videos on the strain field and deformations are submitted as supplemental materials.

The global deformation of the LV can be described as radial contraction, longitudinal shortening and torsion along the LA. The longitudinal strain in the middle ventricle is negative. Circumferential strains reveal larger contraction near the endocardium than near the epicardium. In the circumferential and longitudinal strain fields, we can observe that a high strain area starts from the apical endocardium and passes quickly toward the base, which can be explained by the activation of myocardium. The radial strain in the middle ventricle is 

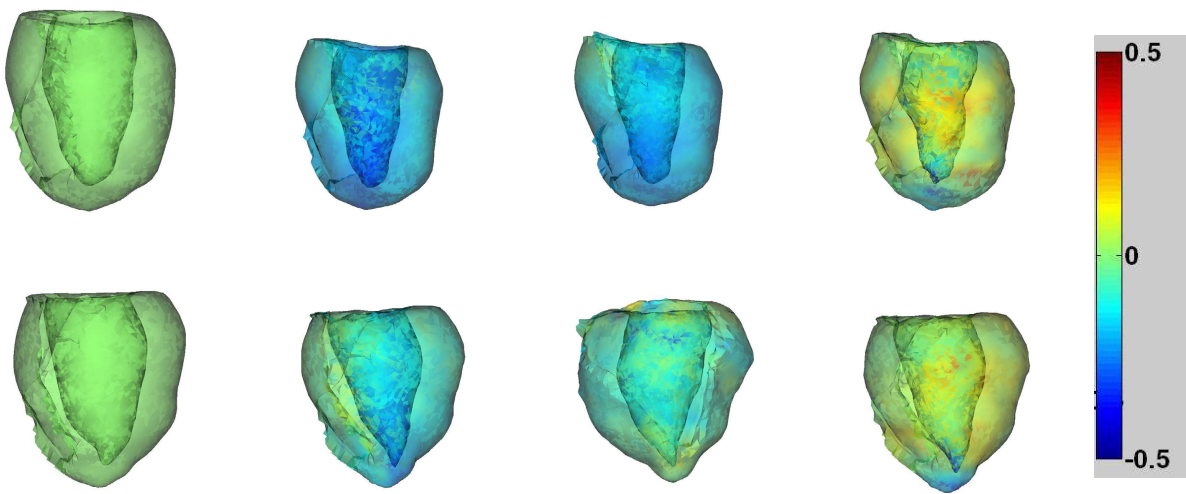

Fig. 3. The first row is a healthy heart and the second row is a hypertrophic heart. The first column shows the LV shape at the end of diastolic, The rest columns show the LV at the end of systolic. The colors show the initial strain, circumferential strain, longitudinal strain and radial strain at the end of systolic from left to right.

mostly positive. Due to the fact that there is less tag information along the radial direction, the strain obtained along the radial direction is less reliable than the other two directions.

We divide each LV into 17 parts and compute the average strain of each part. From the strain time series in the middle anterior calculated from 5 subjects in each group shown in Figure 4, we observe that a healthy heart contracts early in a cardiac cycle, and holds for a short period of time at the end of systolic before it relaxes. The magnitude of the strain in a hypertrophic heart is smaller than a normal heart. The motion of a hypertrophic heart is much slower, hence the contraction and relaxation procedure almost last for a whole cardiac cycle. The tense stage at the end of systolic is not as clear as a normal heart either.
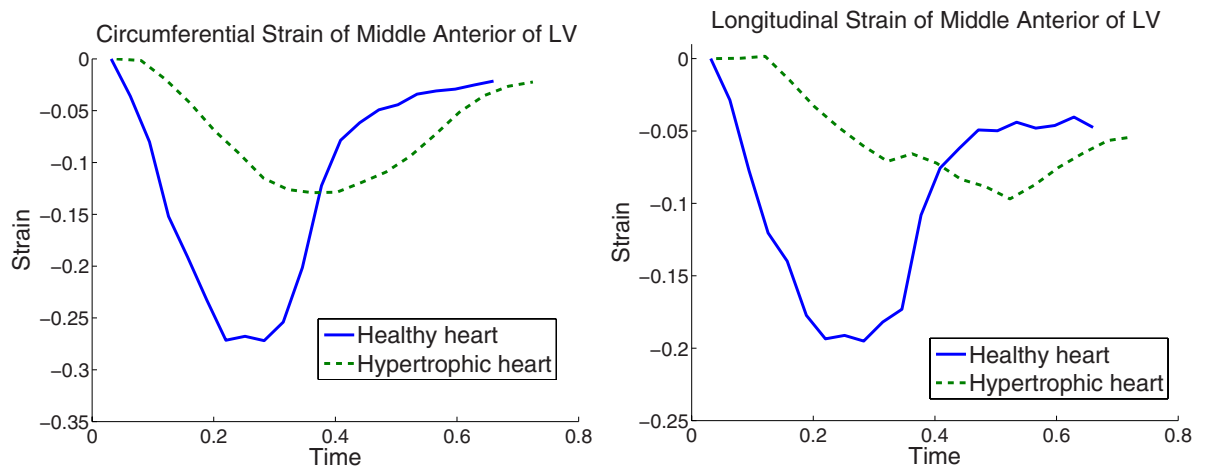

Fig. 4. The strain of the middle anterior of a healthy heart and hypertrophic heart. (a) Circumferential strain (b)Longitudinal strain. 

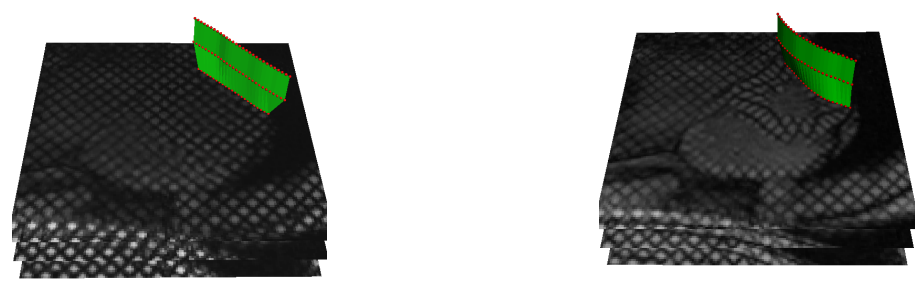

Fig. 5. (a) is a tag plane in the initial state when the LV is at the end of diastolic. (b) is a tag plane at the end of systolic.

\subsection{Validation}

In meshless deformable models, we can track the deformation of an arbitrary point inside the myocardium. We tracked the deformation of a tagging plane with our model for a whole cardiac cycle and projected the tagging plane onto the tMRI at the end of systolic. The projections of the deformed tagging plane align very well with the tagging lines in images. We evaluate the difference between the projections and the tagging lines on 156 tag planes, the MAE is $1.383 \mathrm{~mm}$ (The pixel spacing in tMRI is $1.0938 \mathrm{~mm}$ ). The projections lie in the $95 \%$ confidence interval of the semi-automatically tracked tagging line ground truth. This experiment demonstrated that our model can reconstruct the 3D deformation field accurately.

\section{Conclusion}

We have proposed a meshless deformable model for in vivo LV 3D motion tracking and strain analysis based on tMRI. The model can fully recover the 3D deformation of LV with sparse tMRI slices, while keep the intrinsic geometric details of the myocardium. The meshless approach avoids tedious remeshing procedures in mesh based approaches. The experiments prove the strength of the model against motion complexity, image artifacts, and noises. The strain analysis based on this model can help early diagnosis of cardiac deceases like hypertrophic cardiomyopathy.

\section{References}

1. Huang, J., Abendschein, D., Davila-Roman, V., Amini, A.: Spatio-temporal tracking of myocardial deformations with a $4 \mathrm{D}$ b-spline model from tagged MRI. IEEE Transactions Medical Imaging 18, 957-972 (1999)

2. Declerck, J., Feldmar, J., Ayache, N.: Definition of a 4D continuous planispheric transformatin for the tracking and the analysis of the LV motion. Medical Image Analysis, 197-213 (1998)

3. Jr, T., McVeigh, E.: Model-free reconstruction of three-dimensional myocardial strain from planar tagged $\mathrm{mr}$ images. Journal Magnetic Resonance Imaging 7, 799-810 (1997) 
4. Young, A.: Model tags: direct 3d tracking of heart wall motion from tagged magnetic resonance images. Medical Image Analysis, 361-372 (1999)

5. Park, J., Metaxas, D., Axel, L.: Volumetric deformable models with parameter functions: A new approach to the 3D motion analysis of the LV from MRI-SPAMM. In: ICCV, pp. 700-705 (1995)

6. Haber, E., Metaxas, D.N., Axel, L.: Motion analysis of the right ventricle from the MRI images. In: Wells, W.M., Colchester, A.C.F., Delp, S.L. (eds.) MICCAI 1998. LNCS, vol. 1496, pp. 177-188. Springer, Heidelberg (1998)

7. Park, K., Metaxas, D.N., Axel, L.: A finite element model for functional analysis of 4D cardiac-tagged MR images. In: Ellis, R.E., Peters, T.M. (eds.) MICCAI 2003. LNCS, vol. 2878, pp. 491-498. Springer, Heidelberg (2003)

8. Metaxas, D.N., Terzopoulos, D.: Dynamic 3D models with local and global deformations: Deformable superquadrics. IEEE Transaction on Pattern Analysis Machine Intelligence 13(7), 703-714 (1991)

9. Qian, Z., Metaxas, D.N., Axel, L.: Boosting and noparametric based tracking of tagged MRI cardiac boundaries. In: Larsen, R., Nielsen, M., Sporring, J. (eds.) MICCAI 2006. LNCS, vol. 4190, pp. 636-644. Springer, Heidelberg (2006)

10. Chen, T., Chung, S., Axel, L.: 2D motion analysis of long axis cardiac tagged MRI. In: Ayache, N., Ourselin, S., Maeder, A. (eds.) MICCAI 2007, Part II. LNCS, vol. 4792, pp. 469-476. Springer, Heidelberg (2007)

11. Chen, T., Chung, S., Axel, L.: Automated tag tracking using gabor filter bank, robust point matching, and deformable models. In: Sachse, F.B., Seemann, G. (eds.) FIHM 2007. LNCS, vol. 4466, pp. 22-31. Springer, Heidelberg (2007)

12. Sorkine, O., Lipman, Y., Cohen-Or, D., Alexa, M., Rössl, C., Seidel, H.P.: Laplacian surface editing. In: Proceedings of the Eurographics/ACM SIGGRAPH Symposium on Geometry Processing, pp. 179-188. Eurographics Association (2004)

13. Desbrun, M., Meyer, M., Schroder, P., Barr, A.H.: Implicit fairing of irregular meshes using diffusion and curvature flow. In: ACM SIGGRAPH, pp. 317-324 (1999)

14. Lancaster, P., Salkauskas, K.: Surfaces generated by moving least squares methods. In: Mathematics of Computation, pp. 141-158 (1981) 